\title{
Isotope production for medical usage using fast neutron reactions
}

\author{
By Y. Hatsukawa*, Y. Nagai, T. Kin, M. Segawa, H. Harada, O. Iwamoto, N. Iwamoto, K. Ochiai, K. Takakura, C. Konno \\ and M. Hashimoto
}

Japan Atomic Energy Agency, Tokai, Ibaraki 319-1195, Japan

(Received December 17, 2009; accepted in revised form December 20, 2010)

\section{${ }^{99 m} \mathrm{Tc} /{ }^{99} \mathrm{Mo} / 14-\mathrm{MeV}$ neutrons $/{ }^{99} \mathrm{Mo} /{ }^{9 m} \mathrm{Tc}$ generator / Growth curve / Elution curve}

Summary. ${ }^{99}$ Mo was produced by the ${ }^{100} \operatorname{Mo}(n, 2 n){ }^{99}$ Mo reaction using 14-MeV neutrons from the $\mathrm{D}\left({ }^{3} \mathrm{H}, n\right)^{4} \mathrm{He}$ reaction at Fusion Neutronics Source Facility (FNS) at JAEA.

A target of titanium oxide gel with molybdenum oxide was irradiated and used as the ${ }^{99 \mathrm{~m}} \mathrm{Tc} /{ }^{99} \mathrm{Mo}$ generator.

The growth curve of ${ }^{99 \mathrm{~m}} \mathrm{Tc}$ in the titanium gel target and the elution curve of ${ }^{99 \mathrm{~m}} \mathrm{Tc}$ from the ${ }^{99} \mathrm{Mo} /{ }^{99 \mathrm{~m}} \mathrm{Tc}$ generator were obtained.

\section{Introduction}

${ }^{99 \mathrm{~m}} \mathrm{Tc}$ is the most important radioisotope used in nuclear medicine. The present global demand for ${ }^{99} \mathrm{Mo}$ is about $450 \mathrm{TBq} /$ week which is mostly produced by using only five nuclear reactors in Canada, Belgium, France, Netherlands and South Africa. In 2007, the unanticipated closure of a nuclear reactor in Canada for five weeks reinforced concerns about a reliable long-term supply of ${ }^{99} \mathrm{Mo}$. Note that the reactors mentioned above range in age from 42 to 51 years. Also another problem is that $95-99 \%$ of all ${ }^{99} \mathrm{Mo}$ is produced by the irradiation of highly enriched uranium targets. In order to guarantee safe and reliable supply of ${ }^{99} \mathrm{Mo}$, IAEA recommends the use of the ${ }^{98} \operatorname{Mo}(n, \gamma){ }^{99}$ Mo reaction. In JAEA, fundamental research of the ${ }^{98} \operatorname{Mo}(n, \gamma)^{99}$ Mo reaction was carried out [1] and there are also plans to produce ${ }^{99}$ Mo at the JMTR, one of the research reactors of JAEA.

Recently, two of the present authors proposed a new route to produce ${ }^{99} \mathrm{Mo}$ by the ${ }^{100} \mathrm{Mo}(n, 2 n){ }^{99}$ Mo reaction [2], which has the following characteristic features. First, the reaction cross section is large, about 1.5 barn at $12<E_{\mathrm{n}}<$ $17 \mathrm{MeV}$ [3], which is 10 times larger than the thermalneutron capture cross section of ${ }^{98} \mathrm{Mo}$ [4] (see Fig. 1). Second, the cross sections of the $(n, \alpha),(n, n p)$, and $(n, p)$ reactions are less than a few mb at $E_{\mathrm{n}}=14-\mathrm{MeV}$ (see Fig. 1 ). Third, a large amount of ${ }^{100} \mathrm{Mo}$ target materials can be used, compared to that for proton beam irradiation on ${ }^{100} \mathrm{Mo}$. Fourth, intense neutrons with energies of $12-17 \mathrm{MeV}$ are already available. In the present work we irradiated a target of titanium oxide gel with molybdenum oxide for 6 hours at

*Author for correspondence

(E-mail: hatsukawa.yuichi@jaea.go.jp).

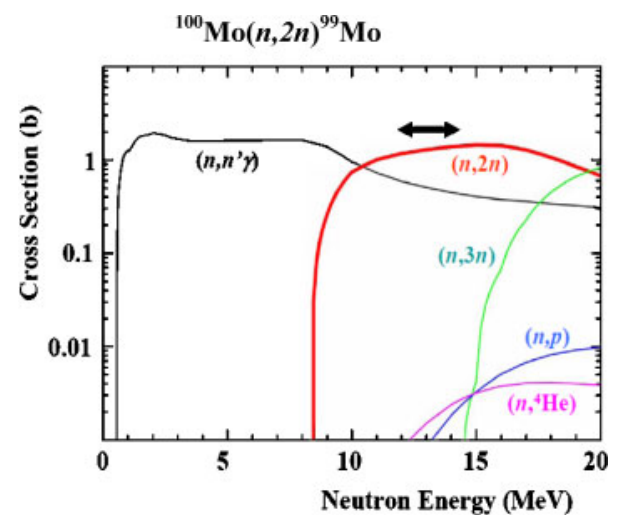

Fig. 1. Excitation functions of ${ }^{100} \mathrm{Mo}$ isotope with fast neutrons obtained from JENDL-3.3. An arrow shows energy region of neutrons from the FNS.

Fusion Neutronics Source Facility (FNS) [5] at JAEA. The neutron flux was about $10^{12} \mathrm{n} / \mathrm{cm}^{2} \mathrm{~s}$ and $10^{7} \mathrm{n} / \mathrm{cm}^{2} \mathrm{~s}$ at the target position.

Technetium-99m ions separated from ${ }^{99} \mathrm{Mo}$ were obtained from the tentative ${ }^{99 \mathrm{~m}} \mathrm{Tc} /{ }^{99} \mathrm{Mo}$ generator which is made from a mixture of titanium gel with molybdenum oxide irradiated with fast neutrons. Monroy-Guzman [6] reported that the ${ }^{99 \mathrm{~m}} \mathrm{Tc} /{ }^{99}$ Mo generator was made from titanium oxide gel with molybdenum oxide which was irradiated with thermal neutrons at a reactor. We tried to make $\mathrm{a}^{99 \mathrm{~m}} \mathrm{Tc} /{ }^{99} \mathrm{Mo}$ generator from titanium oxide gel with molybdenum which irradiated with $14-\mathrm{MeV}$ neutrons.

\section{Experimental}

\subsection{Target preparation}

$1 \mathrm{ml}$ of Titanium(IV) tetrabutoxide $\left(\mathrm{Ti}\left[\mathrm{O}\left(\mathrm{CH}_{2}\right)_{3} \mathrm{CH}_{3}\right]_{4}\right.$ and 0.1-mol of Ammonium molybdate $\left(\mathrm{NH}_{4}\right)_{2} \mathrm{MoO}_{4}$ were dissolved with 10 -ml $n$-Butyl alcohol. In this $n$-Butyl alcohol solution, 1-ml of $0.1-\mathrm{N}^{-} \mathrm{HNO}_{3}$ was dropped slowly while stirring the solution. Soon after dropping the $0.1 \mathrm{~N}-\mathrm{HNO}_{3}$, Butyl titanate began to precipitate. The precipitation, which was Butyl titanium oxide including molybdenum oxide, was washed thoroughly with acetone, and then separated by centrifuge. After drying, the titanium gel powder was pressed into a $\varnothing=10$-mm pellet weighting 500 -mg which included about 30-mg of molybdenum. The pellet was used as a fast neutron irradiation target. A $0.1-\mathrm{mm}$ thick of Niobium foils 
with $\varnothing=10$-mm were positioned in front and behind each pellet as neutron flux monitors.

\subsection{Irradiation at FNS}

A titanium gel target was irradiated with $14-\mathrm{MeV}$ neutrons at the Fusion Neutronics Source (FNS) facility in JAEA, Tokai. The FNS facility is an intense D-T neutron source for the fusion neutronics studies related to a D-T fueled reactor. Since the first D-T neutron production in 1981, which was one of the most intense D-T neutron sources, several experimental programs have been conducted to support ITER design activity. Two beam lines at FNS, the $0^{\circ}$ beam line and the $80^{\circ}$ beam line generate $14-\mathrm{MeV}$ neutrons at rate of $7.2 \times 10^{12} \mathrm{n} / \mathrm{s}$ and $2 \times 10^{11} \mathrm{n} / \mathrm{s}$, respectively. This experiment was performed at the $80^{\circ}$ beam line. The target was placed $10 \mathrm{~cm}$ from the neutron source, and was irradiated for $6 \mathrm{~h}$. The averaged neutron flux of the target position calculated by a Niobium flux monitor was $2.5 \times 10^{7} \mathrm{n} / \mathrm{s}$.

\subsection{Gamma ray measurements}

After $18 \mathrm{~h}$ cooling, gamma rays from the titanium gel target were measured with a Ge detector for $15 \mathrm{~min}$. The irradiated titanium target was dissolved by 2-ml of pure water and the eluted solution was filtrated and dried. Then gamma ray measurements of elution samples were carried out.

\subsection{Growth curve and elution curve}

In order to obtain growth curve of $143-\mathrm{keV}$ gamma rays emitted from ${ }^{99 \mathrm{~m}} \mathrm{Tc}$, gamma ray measurements of the titanium gel were taken every $10000 \mathrm{~s}$ for about $30 \mathrm{~h}$ after the titanium gel was washed with water to remove ${ }^{99 \mathrm{~m}} \mathrm{Tc}$. Then the titanium gel was put in a glass tube. The inner diameter of the glass tube was 5-mm. The titanium gel was retained with quartz wool at the bottom end. Pure water was poured to the titanium gel column to make an elution curve of ${ }^{99 \mathrm{~m}} \mathrm{Tc}$. Every 5 drops of eluent solution were collected on stainless steel plates, and dried for gamma ray measurements. The average volume of 5 drops was $0.27-\mathrm{ml}$.

\section{Results and discussions}

The gamma spectra obtained from the irradiated titanium oxide gel with molybdenum is shown in Fig. 2. In this spectrum, Gamma rays emitted from ${ }^{99 \mathrm{~m}} \mathrm{Tc}$ and ${ }^{99} \mathrm{Mo}$ can be observed. A gamma peak at $143-\mathrm{keV}$ is assigned to ${ }^{99 \mathrm{~m}} \mathrm{Tc}$ and those at 181, 366, 736 and $778-\mathrm{keV}$ to ${ }^{99} \mathrm{Mo}$. Other gamma rays are emitted from $\mathrm{Sc}$ isotopes which are produced by the reaction with Ti isotopes. Gamma rays of 175, 983, 1037 and $1312-\mathrm{keV}$ were emitted from ${ }^{48} \mathrm{Sc}, 159-\mathrm{keV}$ from ${ }^{47} \mathrm{Sc}$ and 889 and $1120-\mathrm{keV}$ from ${ }^{46} \mathrm{Sc}$. Scandium isotopes detected in this spectrum were produced by the ${ }^{48} \mathrm{Ti}(n, p)^{48} \mathrm{Sc}$, ${ }^{47} \mathrm{Ti}(n, p){ }^{47} \mathrm{Sc},{ }^{46} \mathrm{Ti}(n, p){ }^{46} \mathrm{Sc}$ reactions.

Fig. 3 shows a gamma spectrum obtained from the dried elution of the titanium gel target. In this spectrum, mainly $143-\mathrm{keV}$ gamma rays from ${ }^{99 \mathrm{~m}} \mathrm{Tc}$ were observed. No gamma peak of Sc isotopes can be seen in this spectrum. Some small peaks of ${ }^{99} \mathrm{Mo}$, such as those of $181,366,739$ and $778-\mathrm{keV}$, however were also observed. This means some molybdenum

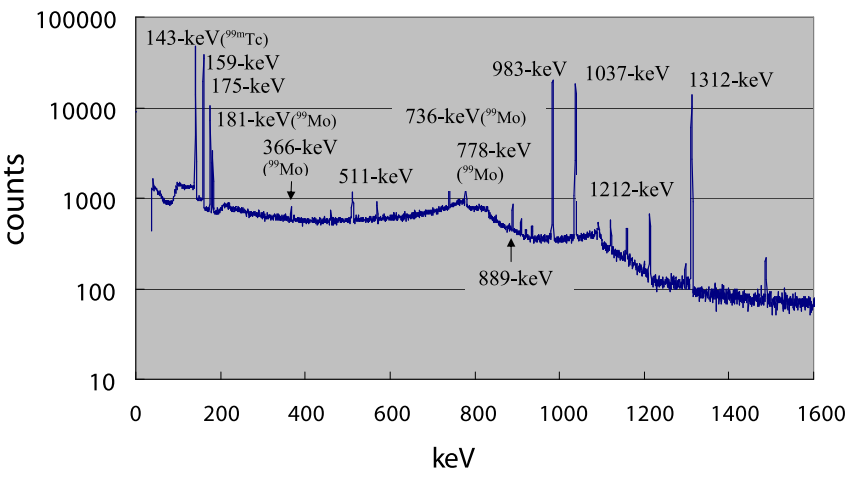

Fig. 2. Gamma ray spectrum obtained from the titanium oxide gel with molybdenum oxide target irradiated with $14-\mathrm{MeV}$ neutron beam at the FNS. Gamma rays emitted from ${ }^{99 \mathrm{~m}} \mathrm{Tc}$ and ${ }^{99} \mathrm{Mo}$ can be observed. A gamma peak at $143-\mathrm{keV}$ is assigned to ${ }^{99 \mathrm{~m}} \mathrm{Tc}$ and those at 181 , 366, 736 and $778-\mathrm{keV}$ to ${ }^{99} \mathrm{Mo}$. Gamma rays of $175,983,1037$ and $1312-\mathrm{keV}$ were emitted from ${ }^{48} \mathrm{Sc}, 159-\mathrm{keV}$ from ${ }^{47} \mathrm{Sc}$ and 889 and $1120-\mathrm{keV}$ from ${ }^{46} \mathrm{Sc}$.

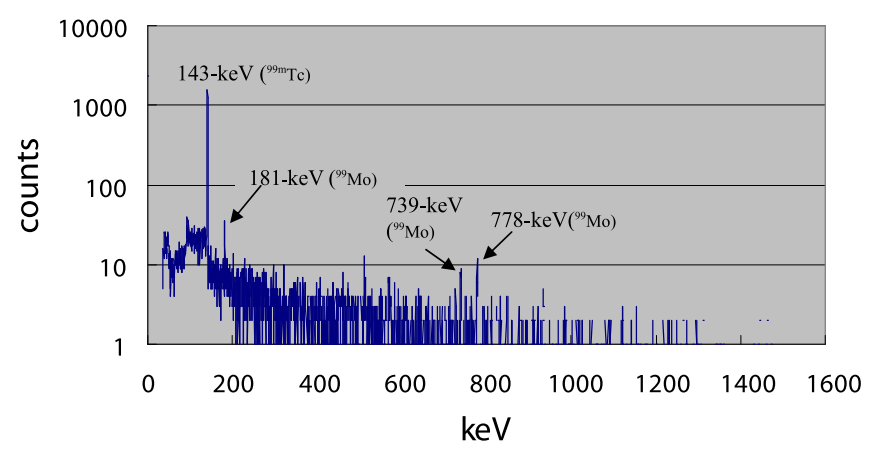

Fig. 3. Gamma ray spectrum obtained from the elution of the titanium oxide gel with molybdenum oxide target. Only $143-\mathrm{keV}$ gamma ray from ${ }^{99 \mathrm{~m}} \mathrm{Tc}$ was mainly observed. Other gamma rays of $181,366,736$ and $778-\mathrm{keV}$ were assigned to ${ }^{99} \mathrm{Mo}$.

isotopes leaked from the titanium gel. However this problem could be improved by attaching a small $\mathrm{Al}_{2} \mathrm{O}_{3}$ column at the end of the titanium gel column directly. Mo isotopes leaked from the titanium gel column will be efficiently adsorbed into the $\mathrm{Al}_{2} \mathrm{O}_{3}$ column, then, only technetium isotopes can pass though both columns.

After the titanium gel was washed with water in order to remove ${ }^{99 \mathrm{~m}} \mathrm{Tc}$, gamma ray measurements of the titanium gel were carried out for about $30 \mathrm{~h}$. The growth curve of the intensity of 143-keV gamma rays was obtained (see Fig. 4). The observed gamma ray intensities of ${ }^{99 \mathrm{~m}} \mathrm{Tc}$ are shown by closed circles. Two theoretical growth curved are also shown in Fig. 4.

The theoretical curves are obtained from the following equation.

$$
N_{1}=\lambda_{1} N_{0}\left(\frac{e^{-\lambda_{1} t}}{\lambda_{2}-\lambda_{1}}+\frac{e^{-\lambda_{2} t}}{\lambda_{2}-\lambda_{1}}\right)+N_{1}^{0} e^{-\lambda_{2} t}
$$

where $N$ and $\lambda$ represent number of atoms and decay constants, and subscript 0 and 1 denote ${ }^{99} \mathrm{Mo}$ and ${ }^{99 \mathrm{~m}} \mathrm{Tc}$, respectively, $N_{1}^{0}$ the number of atoms of ${ }^{99 \mathrm{~m}} \mathrm{Tc}$ at $t=0$.

The solid line is obtained by assuming that no ${ }^{99 \mathrm{~m}} \mathrm{Tc}$ at the titanium gel was washed with water $\left(N_{1}^{0} e^{-\lambda_{2} t}=0\right.$ at $t=0)$. 


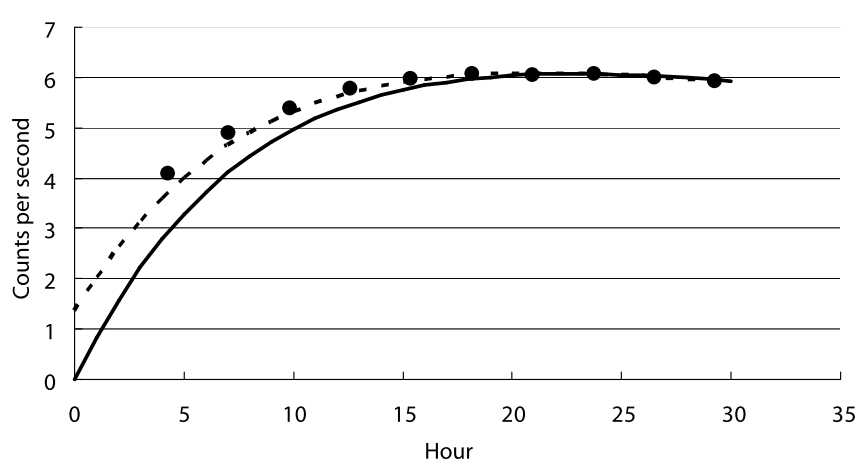

Fig. 4. Growth curve of $143-\mathrm{keV}$ gamma ray from ${ }^{99 \mathrm{~m}} \mathrm{Tc}$ in the titanium oxide gel with molybdenum oxide which was washed by water. Closed circles show counting rates of $143-\mathrm{keV}$ gamma rays from ${ }^{99 \mathrm{~m}} \mathrm{Tc}$. The solid line shows theoretical growth curve of $143-\mathrm{keV}$ gamma rays with assuming ${ }^{99 \mathrm{~m}} \mathrm{Tc}=0$ at $t=0$. The broken line shows theoretical growth curve of $143-\mathrm{keV}$ gamma rays, assuming half value of ${ }^{99 \mathrm{~m}} \mathrm{Tc}$ remained in the titanium gel at $t=0$.

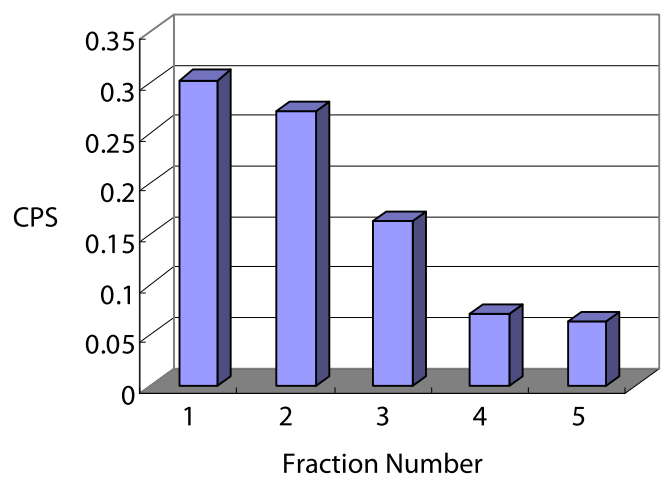

Fig. 5. Elution curve of ${ }^{99 \mathrm{~m}} \mathrm{Tc}$ from titanium oxide gel with molybdenum oxide column. The $143-\mathrm{keV}$ gamma ray intensity of ${ }^{99 \mathrm{~m}} \mathrm{Tc}$ from each fraction was plotted. Each fraction contains 5 drops which is about $0.27-\mathrm{ml}$.

The broken line is obtained by assuming that half number of ${ }^{99 \mathrm{~m}} \mathrm{Tc}$ of maximum value were remained at $t=0$.

Observed results are agreed with the broken line than the solid line. This means after water rinsing, about half of ${ }^{99 \mathrm{~m}} \mathrm{Tc}$ were still remained in the titanium gel in this conditions.

The elution curve of ${ }^{99 \mathrm{~m}} \mathrm{Tc}$ obtained from the titanium oxide gel with molybdenum oxide is shown in Fig. 5. Five fragment samples were made and gamma ray measurements were carried out. Each fragment contains 5 drops which is about $0.27-\mathrm{ml}$. The obtained intensities of $143-\mathrm{keV}$ gamma rays from ${ }^{99 \mathrm{~m}} \mathrm{Tc}$ were plotted in Fig. 5. This result shows more than $80 \%{ }^{99 \mathrm{~m}} \mathrm{Tc}$ were collected from first 3 fractions which contained $0.81 \mathrm{ml}$.

\section{Summary}

In this study, ${ }^{99} \mathrm{Mo}$ was effectively produced by the ${ }^{100} \mathrm{Mo}(n, 2 n){ }^{99} \mathrm{Mo}$ reaction using $14-\mathrm{MeV}$ neutrons from the FNS facility at JAEA. A target of titanium oxide gel with molybdenum oxide was irradiated and used as the ${ }^{99 \mathrm{~m}} \mathrm{Tc} /{ }^{99}$ Mo generator. From the titanium oxide gel generator, ${ }^{99 \mathrm{~m}} \mathrm{Tc}$ was separated from Mo target. Although some of ${ }^{99} \mathrm{Mo}$ was found in the ${ }^{99 \mathrm{~m}} \mathrm{Tc}$ fraction, this leakage can be prevented by using an $\mathrm{AlO}_{2}$ column. Recent developments of large scale fast neutron sources, such as IFMIF [7] and SPRIL-2 [8], will encourage the production of ${ }^{99 \mathrm{~m}} \mathrm{Tc}$ by the ${ }^{100} \mathrm{Mo}(n, 2 n){ }^{99}$ Mo reaction.

\section{References}

1. Shikata, E., Iguchi, A.: Production of ${ }^{99}$ Mo and its application in nuclear medicine. J. Radioanal. Nucl. Chem. 102, 533-550 (1986).

2. Nagai, Y., Hatsukawa, Y.: Production of ${ }^{99}$ Mo for nuclear medicine by ${ }^{100} \mathrm{Mo}(n, 2 n)^{99}$ Mo. J. Phys. Soc. Jpn. 78, 033201 (2009).

3. Shibata, K, Kawano, T,Nakagawa, T.,Iwamoto, O., Katakura, J., Fukahori, T., Chiba, S., Hasegawa, A., Murata, T., Matsunobu, H., Ohsawa, T., Nakajima, Y., Yoshida, T., Zukeran, A., Kawai, M., Baba, M., Ishikawa, M., Asami, T., Watanabe, T., Watanabe, Y., Igashira, M., Yamamuro, N., Kitazawa, H., Yamano, N., Takano, H.: Japanese Evaluated Nuclear Data Library Version 3 Revision-3: JENDL-3.3. J. Nucl. Sci. Technol. 39, 1125-1136 (2002).

4. Firestone, R. B., Shirley, V. S.: Table of Isotopes. $8^{\text {th }}$ Edn., John Wiley \& Sons (1995).

5. Nakamura, T., Maekawa, H.,Ikeda, Y., Oyama, Y.: A DT Neutron Source for Fusion Neutronics Experiments at the JAERI. Proc. Int. Ion Engineering Congress, Kyoto, Japan, 12-16 September 1983, Institute of Electrical Engineers of Japan (1983), p. 567.

6. Monroy-Guzman, F., Cortes Romero, O., Diaz Velazquez, H.: Titanium molybdate gels as matrix of ${ }^{99} \mathrm{Mo} /{ }^{99 \mathrm{~m}} \mathrm{Tc}$ generators. J. Nucl. Radiochem. Sci. 8, 11-19 (2007).

7. Martone, M.: IFMIF Conceptual Design Activity, Final Report, IFMIF CDA Team, ENEA Frascati Report, RT/ERG/FUS/96/11 (1996).

8. Ledoux, X., Aiche, M., Ban, G., Barreau, G., Baumann, P., Bem, P., Blideanu, V., Blomgren, J., Czajkowski, S., Dessagne, P., Dupont, E., Dore, D., Ethvignot, T., Fischer, U., Gunsing, F., Jacquot, B., Jurado, B., Kerveno, M., Lecolley, F. R., Lecouery, J. L., Negoita, F., Obertedt, S., Petrascu, M., Plompen, A. J. M., Rejmund, F., Ridikas, D., Rudolf, G., Shcherbakov, O., Simakov, S. P., Taieb, J.: A neutron beam facility at SPIRAL-2, International Conf. on Nucl. Data for Science and Technology 2007 (2008), pp. 459-462. 